\title{
Erratum: Quantum Stress Focusing in Descriptive Chemistry [Phys. Rev. Lett. 100, 206405 (2008)]
}

Jianmin Tao, Giovanni Vignale, and I. V. Tokatly

(Received 16 October 2015; published 9 November 2015)

DOI: 10.1103/PhysRevLett.115.209901

PACS numbers: 71.15.Mb, 31.15.E-, 71.45.Gm, 99.10.Cd

There is a sign error in the last terms of Eqs. (15) and (16). They should read

$$
\begin{gathered}
p_{i j}^{\mathrm{XC}}=\delta_{i j}\left(n v_{\mathrm{XC}}-e_{\mathrm{XC}}\right)+\frac{\partial_{i} n \partial_{j} n}{2 k_{F} n|\nabla n|} \frac{\partial e_{\mathrm{XC}}}{\partial s}, \\
p^{\mathrm{XC}}=n v_{\mathrm{XC}}-e_{\mathrm{XC}}+\frac{s}{3} \frac{\partial e_{\mathrm{XC}}}{\partial s} .
\end{gathered}
$$

This error slightly modifies the shell structure of the atoms, as shown in Fig. 1, follows:
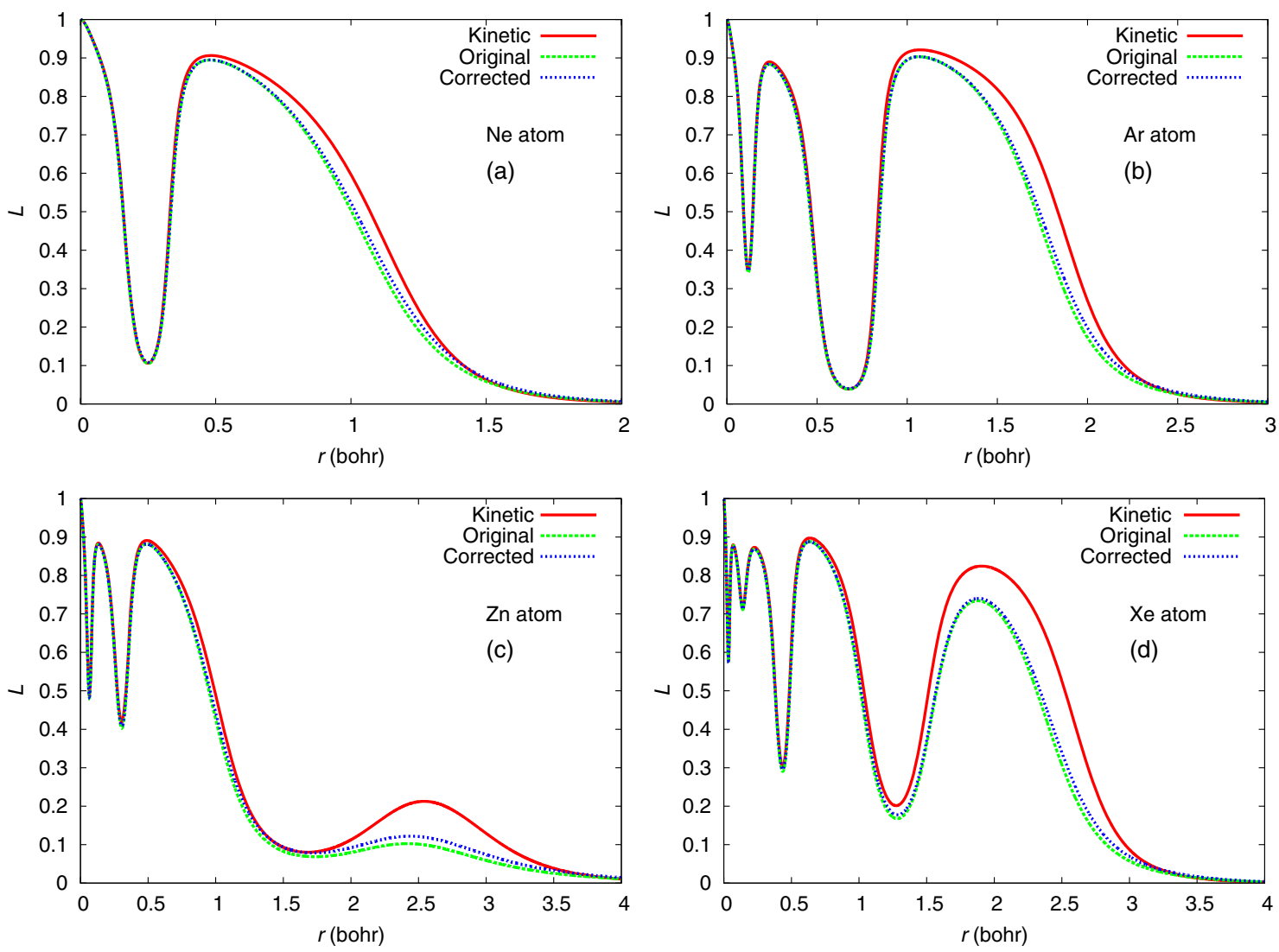

FIG. 1 (color online). Atomic shell structure from the original quantum pressure formula and the corrected Eqs. (15) and (16). 